\title{
Target guiding self-avoiding random walk with intersection algorithm for minimum exposure path problem in wireless sensor networks
}

Tinghong Yang ${ }^{1,2+}$, Dali Jiang ${ }^{1 *}$ D, Haiyang Fang ${ }^{1 \dagger}$, Mian Tan ${ }^{1}$, Li Xie $^{3}$ and Jing Zhao ${ }^{2^{*}}$

\begin{abstract}
To solve minimum exposure path (MEP) problem in wireless sensor networks more efficiently, this work proposes an algorithm called target guiding self-avoiding random walk with intersection (TGSARWI), which mimics the behavior of a group of random walkers that seek path to their destinations in a strange area. Target guiding leads random walkers move toward their end points, while self-avoiding prevents them from taking roundabout routes. Route intersections further accelerate the speed of seeking connected paths. Dijkstra algorithm (DA) is applied to solve MEP problem in a sub-network formed by multiple connected paths that walkers generate (called TGSARWI DA). Simulations show that the path exposure found by TGSARWI DA is very close to that by DA in the global network (Global DA), whereas the time complexity of computation is much lower. Compared with existing heuristic algorithms such as physarum optimization algorithm (POA), our algorithm shows higher generality and efficiency. This algorithm also exhibits good robustness to the fluctuations of parameters. Our algorithm could be very useful for the solution to MEP problem in fields with large- or highdensity sensors.
\end{abstract}

Keywords: Minimum exposure path, Target guiding, Self-avoiding random walk, Intersection, Time complexity of computation, Robustness

\section{Introduction}

Wireless sensor network (WSN) is a kind of distributed sensing networks, whose nodes can detect the surrounding environment [1-3]. Given the characteristics of large-scale deployment, easy extensibility, and low price, WSN has been widely applied to such fields as military, target tracking, intelligent transportation, environmental monitoring, and health care [4-11]. The research and application of wireless sensor networks have attracted massive attention.

Coverage is a vital index to evaluate the quality of service (QoS) of WSN's sensing function [12-16]. According to various characteristics of monitored objects, coverage can

\footnotetext{
* Correspondence: jiangdali99@163.com; zhaojanne@gmail.com Tinghong Yang and Haiyang Fang contributed equally to this work. 'Equal contributors

${ }^{1}$ Department of Military Logistics, Army Logistics University, Chongqing, China

${ }^{2}$ Department of Mathematics, Army Logistics University, Chongqing, China Full list of author information is available at the end of the article
}

be classified into three types, i.e., area coverage, point coverage, and barrier coverage [17]. The present studies on the measurement of coverage mostly focus on barrier coverage [18-20] and therefore this study on barrier coverage is aimed to find one or various paths connecting source point and target point, and quantitatively describe the sensing quality of mobile objects along the path [21, 22]. The concept of path exposure is used to evaluate coverage quality in the sensor-deployed field with the consideration of path length and exposure simultaneously.

With respect to path exposure, there are two widely divergent viewpoints. One is best-case coverage, which is to find a path with the highest observability. The Maximal Exposure Path and the Maximal Support Path are two solutions to the best-case coverage [23-25]. The other is the worstcase coverage, which is to design a path through the sensor field and make the minimum probability of detecting the mobile object. There exist two well-known approaches with respect to the worst-case coverage, i.e., minimal exposure 
path (MEP) and the maximal breach path [26]. The maximal exposure path and minimal exposure path are essentially the same problem. Hence, we focus on the MEP problem of worst-case coverage in this paper. The MEP problem is the variation of functional extremum in mathematics [27]. Theoretically, it is possible to find the optimum path by solving the corresponding Euler-Lagrange Equation [28]. When there is only one sensor in the detecting region, ref. [29] obtains the exact analytical solution to MEP problem. However, in the multiple-sensor region, higher-order non-linearity makes it difficult to express and solve the Euler-Lagrange equation [29-31]. Some approaches have been proposed to address this problem, such as Voronoi diagram-based approach and grid-based approach. However, the time complexity of computation of those approaches will increase with the rise of the grid scale or sensor density. Therefore, it is essential to propose an algorithm to reduce the time complexity of computation and guarantee the quality of solutions simultaneously.

This paper is aimed to decrease the time complexity of computation of grid-based approach in order to solve MEP problem with large-scale field or high-density sensors. An algorithm is proposed and named as target guiding selfavoiding random walk with intersection (TGSARWI), which mimics the behavior that an individual seeks a path from the source point to the target point in a strange area. In this algorithm, multiple random walkers start from the source point and the target point respectively and head to the opposite sides along edges of the grid network. At each step, each random walker moves to a neighbor chosen according to the edge exposure and the direction to its end point. When routes of walkers from different start points intersect, one or multiple paths will be created between the source and the target, so that a connected sub-network can be formed. Finally, the shortest path algorithm is employed to seek the minimum exposure path in this sub-network.

The main contributions of this paper are summarized as follows:

- Compared with the previous algorithm, TGSARWI DA can solve MEP problem in large-scale field with acceptable complexity.

- The complexity of TGSARWI DA is insensitive to the number of sensors, enabling to solve MEP problem with much higher density sensors.

- Unlike the POA, parameters in TGSARWI DA need no adjustment as the background of MEP problem changes. Hence, the newly proposed algorithm has better adaptability to environment.

The rest of this paper is organized as follows: Section 2 summarizes related approaches about the minimal exposure path problem. Section 3 introduces the model of MEP problem. Section 4 proposes TGSARWI DA algorithm. Section
5 evaluates the proposed TGSARWI DA by extensive simulations.

\section{Related work}

The goal of MEP problem is to find a path through the detecting region with sensor deployment and to ensure the object that moves along the path has the least possibility detected by sensors. When the detecting region is deployed by multiple sensors, the finding of the minimum exposure path, due to the highly ordered nonlinearity of MEP problem, will become rather complex using the Euler-Lagrange equation. Considering the difficulty in obtaining exact solution to the MEP problem, the most popular strategy is to convert the continuous MEP problem to discrete problem. The discrete method includes two principle approximate approaches. One is Voronoi diagram-based approach and the other is grid-based approach.

Voronoi diagram-based approach is proposed in ref. [26]. In this approach, the detecting region is divided into $n$ ( $n$ is the sensor number) polygons according to the geometric positions of sensors, and each polygon is called a Voronoi cell. The distance of any point in a Voronoi cell to its sensor is closer than that to the other sensors. All the edges of the polygons form a graph called Voronoi graph. The weight of the edge is defined as the exposure along the Voronoi edge. The object can move only along the Voronoi edge. Then, the MEP problem is transformed into a shortest path problem and could be solved by the shortest path algorithm. Many improvements are made based on this approach. For example, Djidjev integrates Voronoi diagram and Euler-Lagrange equation to find the minimum exposure path $[29,30]$. Zhang et al. propose a distributed path search method based on Voronoi diagram [32]. In ref. [33], Zhou applies Voronoi graph and Dijkstra algorithm to search vulnerable path in the sensors network. However, Voronoi diagram-based approach cannot be used in networks including directional sensors, unless the range of directional sensor is transformed into a special region. In addition, the time complexity of computation will be sharply increased as the total number of sensors grows. Moreover, this approach is also inaccessible when the source and target are not on the Voronoi edges or the sensing intensity is not decided only by the nearest sensor [34].

Grid-based approach divides detecting region into $m \times n$ square grid cells. Each cell has four nodes and four edges. Each node connects its four neighboring nodes by four edges. The weight of each edge is the exposure, which is obtained by sensing intensity from the sensors. Then, the whole nodes and edges form a weighted undirected network. The object is restricted to move along the edges or diagonals of the grid, and then the minimum exposure path is identified based on the edge exposures [24]. Liu et al. uses 
a grid-based approach in the field with directional and omni-directional sensors deployment, and applies the physarum optimization to obtain the minimum exposure path [35]. Ref. [36] proposes a bond percolation-based scheme to put the exposure path problem into a 3D uniform lattice, and then uses grid-based approach to find the minimum exposure path. Aiming at the MEP problem with path constraint conditions, Hao et al. divides the path into three parts. The paths of the first part and the last part are calculated by grid-based approach, while the middle part is solved through the hybrid genetic algorithm [37]. Liu uses adaptive cell decomposition to transform the minimal exposure path problem into a discrete problem, and then designs an OMEPS algorithm to search for the obstacle-avoidance minimum exposure path in the grid-based network [38]. Accordingly, other various improved algorithms based on grid partition also have been proposed [39-42]. It is noted that, in grid-based approach, the larger the scale of the grid network within a fixed region is, the more accurate the solution to the MEP problem will be. However, since the complexity rises sharply as the grid scale increases, it is difficult to solve the problem in large-scale grids. Some heuristic algorithms have been proposed to simplify the complexity. For example, Liu et al. proposed a physarum optimization algorithm (POA) based on the light-avoidance of physarum, which could be used to obtain minimum exposure path with edgecutting method in the grid [40]. The shortage of this method is that, in the simulations, parameters of POA need to be retuned when the grid scale and sensor density change.

\section{Minimum exposure path problem}

Generally speaking, wireless sensors applied in different fields have different functions, but their common feature is that their detecting sensitivity gradually attenuates as the detecting distance increases. For directional sensor, the sensitivity of the sensor is inversely proportional to the offset angle.

Suppose $n_{s}$ sensors $\left\{s_{1}, s_{2}, \ldots, s_{n_{s}}\right\}$, directional or omnidirectional, are randomly distributed in a rectangle field $Q$ which is an arbitrary point in the sensor-detected field. The detecting sensitivity $s s\left(s_{i}, Q\right)$ of sensor $s_{i}$ to the point $Q$ could be represented as follows:

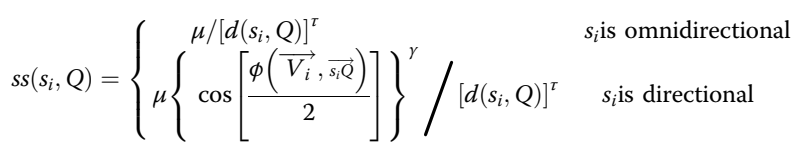

Where $d\left(s_{i}, Q\right)$ represents Euclidean distance between sensor $s_{i}$ and point $Q . \overrightarrow{V_{i}}$ denotes the unit vector which determines the sensing direction of sensor $s_{i}$ in the case of directional sensor. $\overrightarrow{s_{i} Q}$ is the vector from sensor $s_{i}$ to point $Q, \phi\left(\overrightarrow{V_{i}}, \overrightarrow{s_{i} Q}\right)$ denotes the angle between $\overrightarrow{V_{i}}$ and $\overrightarrow{s_{i} Q}$. Parameter $\mu, \tau$, and $\gamma$ are the sensor-dependent parameters. According to the literature [40], parameter $\mu$ denotes the sensitivity of the sensor at the unit distance or the direction of directional sensor, parameter $\tau$ denotes the attenuation coefficient of sensor sensitivity on distance, and parameter $\gamma$ denotes the attenuation coefficient of the directed sensor sensitivity on deviating from the central direction.

The sensitivity $S$ of point $Q$ detected by field sensors could be represented by two methods. One is to consider the sensitivity $S$ as the sum of $n_{s}$ sensors' sensitivities, which can be represented as $S(Q)=\sum_{i=1}^{n_{s}} s s\left(s_{i}, Q\right)$. The other is to consider the sensitivity $S$ as the maximum sensitivity of $n_{s}$ sensors to the point $Q$, which is $S$ $(Q)=\max _{i=1, \ldots, n_{s}} s s\left(s_{i}, Q\right)$. Following ref. [40], we use the latter method to represent the sensitivity.

The concept of exposure is used to measure the coverage quality of the field with sensor deployment. Once the walker's path $f(x)$ is known, the exposure $E(f(x))$ of the path could be calculated by curvilinear integral method as follows:

$$
E(f(x))=\int_{v_{s}}^{v_{e}} S(x, f(x)) \sqrt{1+\left(f^{\prime}(x)\right)^{2}} d x
$$

Where $v_{s}$ and $v_{e}$ are the source point and target point, respectively.

MEP problem is to find a path in which the exposure reaches minimum, while the minimum $E(f(x))$ could be obtained by variation method of extremum. Here, we denote $F=S(x, f(x)) \sqrt{1+\left(f^{\prime}(x)\right)^{2}}$ and substitute it into the following Euler-Lagrange equation:

$$
\frac{\partial F}{\partial f(x)}-\frac{\mathrm{d}}{\mathrm{d} x} \frac{\partial F}{\partial f^{\prime}(x)}=0
$$

Theoretically, the minimum $E(f(x))$ can be obtained only when the above equation is solved. However, in the field with multiple sensors, higher-order nonlinearity makes it difficult to solve the Euler-Lagrange equation. Therefore, the problem of continuous minimum exposure path is transformed into a discrete problem in this paper.

Supposing that the detected field is a rectangle area, the rectangle can be divided into $m \times n$ grids. Then, the walker can move only along the edges of the grid network, as shown in Fig. 1.

Here, the sensor-detected grid is a weighted connected network graph $G=(V, E, W)$, where $V=\left\{v_{1}, v_{2}, \ldots, v_{i}, \ldots\right.$, $\left.v_{m \times n}\right\}$ represents the set of all nodes and $E$ is the set of all edges. Let $w_{i j} \in W$ denote the weight of the edge $e_{i j}$ between two nodes $v_{i}$ and $v_{j}$, which shows the exposure of $e_{i j}$ detected by all sensors in the grid field. Since this graph is an undirected network graph, we have $w_{i j}=w_{j i}$. 


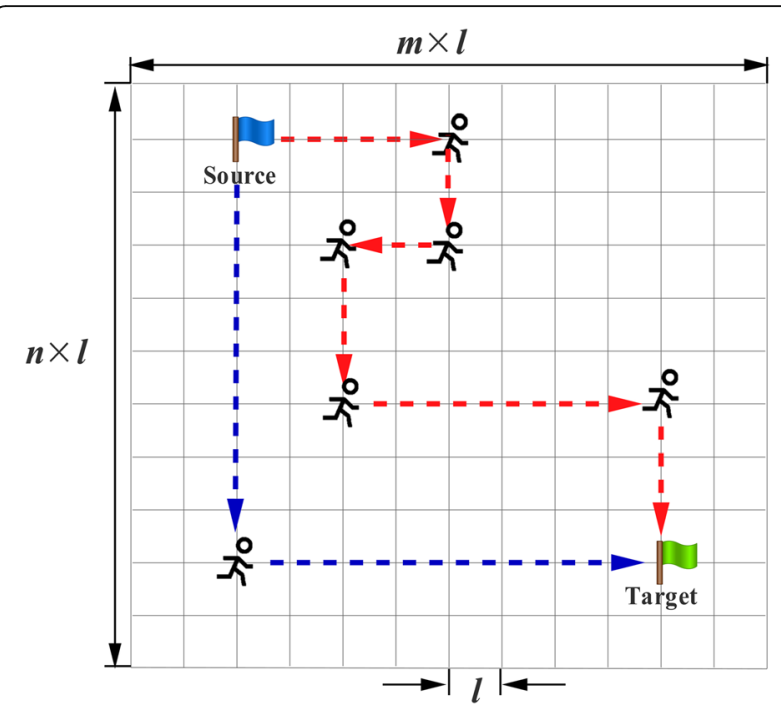

Fig. 1 The grid network with scale $m \times n$, where the walker can move only along its edges

Let the length of the edge be $l$, the coordinate of node $v_{i}$ be $\left(x_{i}, y_{i}\right)$, the weight $w_{i j}$ could be calculated as follows:

$$
\begin{aligned}
w_{i j}= & E\left(e_{i j}\right) \\
= & \begin{cases}\int_{\min \left\{x_{i}, x_{j}\right\}}^{\min \left\{x_{i}, x_{j}\right\}+l} S\left(\left(x, y_{i}\right)\right) d x \quad e_{i j} \text { is horizontal } \\
\int_{\min \left\{y_{i}, y_{j}\right\}}^{\min \left\{y_{i}, y_{j}\right\}+l} S\left(\left(x_{i}, y\right)\right) d y \quad e_{i j} \text { is vertical }\end{cases}
\end{aligned}
$$

Once the exposure of each edge in the grid is obtained, the minimum exposure path between source point $v_{s}$ and target point $v_{e}$ can be got through optimized algorithm (such as Dijkstra algorithm). Nevertheless, the time complexity of Dijkstra algorithm is $O\left(m^{2} n^{2}\right)$, making it difficult to solve MEP problem in a large-scale field. Therefore, this research will apply random walk to imitate the path-finding process of a walker in a strange area. After generating a sufficient number of connected paths between $v_{s}$ and $v_{e}$, Dijkstra algorithm is used to seek minimum exposure path in the sub-network formed by these connected paths.

\section{TGSARWI DA algorithm for MEP problem}

\subsection{Self-avoiding random walk}

For the random walk algorithm [43], a walker starts at the node $v_{i}$ and moves to a randomly chosen neighbor $v_{j} \in V$ at each step. The above process could be denoted as $x^{t+1}$ $=P x^{t}$, where $P$ represents the matrix of transition probability, which is column-normalized matrix of weighted adjacency matrix of network graph $G$. The transition probability from node $v_{i}$ to $v_{j}$ is inversely proportional to the weight $w_{i j}$ of the edge $e_{i j}$, which could be represented as follows:

$$
p_{i j}=\frac{1 / w_{i j}}{\sum_{e_{i k} \in E}\left(1 / w_{i k}\right)}
$$

In a random walk, the node-visiting sequence of the walker is a finite state Markov chain, which is only related to the currently visited node rather than the previous sequence of nodes. However, in the shortest path-finding process, the next node that will be visited should not be included in the nodes that have been visited. Namely, the transfer process of MEP problem is not a Markov chain. Thus, a self-avoiding random walk is defined to avoid any visited nodes. In such a random walk, for a path $H$, the transition probability $p_{i j}^{(1)}(H)$ from node $v_{i}$ to $v_{j}$ is modified as follows:

$$
p_{i j}^{(1)}(H)=\left\{\begin{array}{cc}
\frac{1 / w_{i j}}{\sum_{e_{i k} \in E v_{k} \notin H} 1 / w_{i k}} & v_{j} \notin H \\
0 & v_{j} \in H
\end{array}\right.
$$

Where, the operator " $\&$ " denotes the logical operation symbol "AND". Unfortunately, as shown in Fig. 2, when the walker performs a self-avoiding random walk, it could be besieged by sensors or the previously visited path. In this situation, the walker is expected to take local backtracking or restart a walk.

\subsection{Self-avoiding random walk with intersection}

Since the time complexity of computation of the selfavoiding random walk to find a path between a source point and a target point is the square of the distance, the algorithm of self-avoiding random walk with intersection is proposed.

We assume that the source and target are two "bases," each of which dispatches several "walkers" to contact each other. When the walkers move in the grid network $G$ by self-avoiding random walk, they move by transition probability $p_{i j}^{(1)}(H)$ and leave marks on the nodes that have been visited. If two walkers from two bases meet directly or one walker moves to a node that has been visited by a walker from the opposite base, the path connecting two bases could be generated by routes of these two walkers, as Fig. 3 shows. Thus, this process is called as a self-avoiding random walk with intersection.

Suppose that $n_{u}\left(n_{u} \gg 1\right)$ walkers are simultaneously dispatched from the base $v_{s}$ and $v_{e}$, respectively, and routes created by walkers from the two sides are defined as $H_{i}^{s}(1$ $\left.\leq i \leq n_{u}\right)$ and $H_{j}^{e}\left(1 \leq j \leq n_{u}\right)$. In the seeking process, if $H_{i}^{s} \cap$ $H_{j}^{e} \neq \varnothing$, there exists one intersection between routes from the $i$-th walker from $v_{s}$ and the $j$-th walker from $v_{e}$. Then, these two walkers stop path seeking. Since the walkers 
a

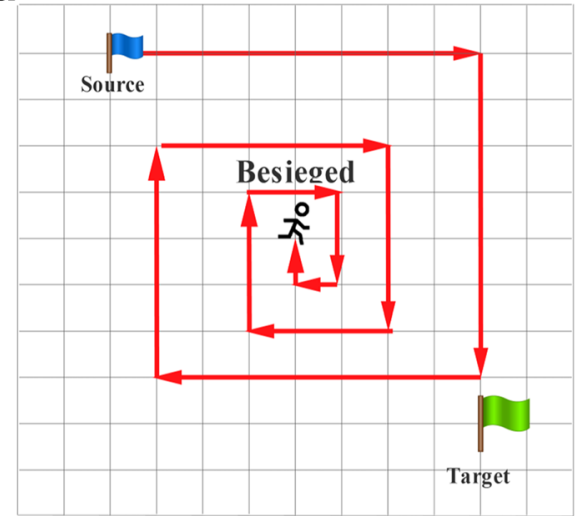

b

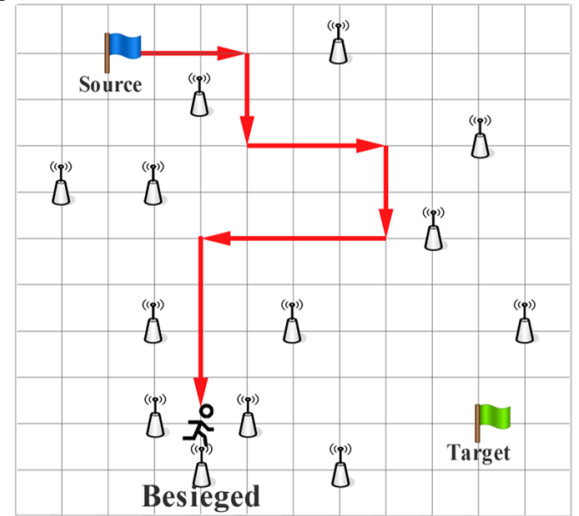

Fig. 2 The diagram of the besieged walker, where a shows the walker is besieged by the path visited before, $\mathbf{b}$ shows the walker is besieged by sensors

from the two sides always check the intersection during the seeking processes, they could ensure that the connected path has only one intersection point. The connected path obtained between $v_{s}$ and $v_{e}$ is described as follows.

Assume that $H_{i}^{s} \cap H_{j}^{e}=\left\{v_{0}\right\}, H_{i}^{s}=\left\{v_{s}, v_{i_{1}}, v_{i_{2}}, \ldots, v_{i_{n}}, v_{0}\right.$, $\ldots\}$, and $H_{j}^{e}=\left\{v_{e}, v_{j_{1}}, v_{j_{2}}, \ldots, v_{j_{n^{\prime}}}, v_{0}, \ldots\right\}$, then the connected path between $v_{s}$ and $v_{e}$ is $H=\left\{v_{s}, v_{i_{1}}, v_{i_{2}}, \ldots, v_{i_{n}}\right.$, $\left.v_{0}, v_{j_{n^{\prime}}}, v_{j_{n^{\prime}-1}}, \ldots, v_{j_{2}}, v_{j_{1}}, v_{e}\right\}$.

\subsection{Target guiding self-avoiding random walk with intersection}

As a single path obtained by random walk possesses great randomness, it probably includes a roundabout route as shown in Fig. 3, which is unacceptable as the shortest path. Thus, target guiding is added in the random walk.

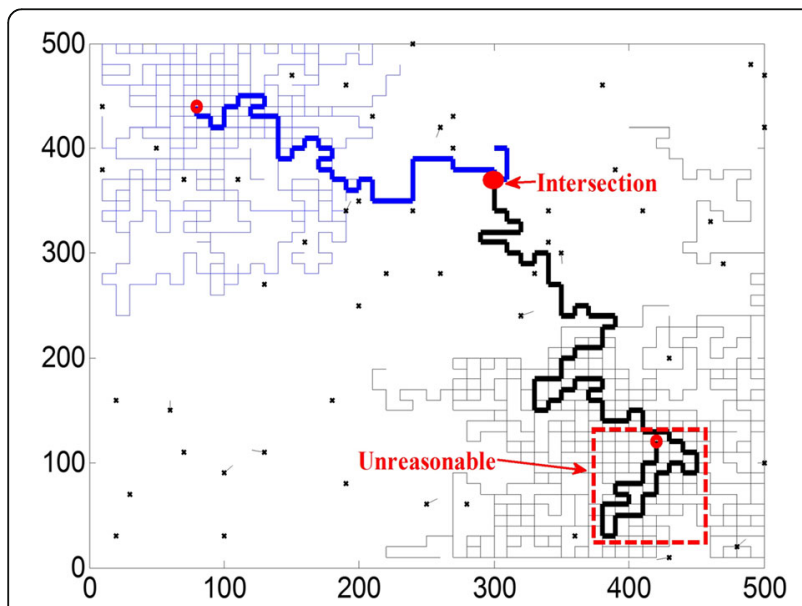

Fig. 3 The diagram of self-avoiding random walk with intersection, where red cycle nodes represent the two bases, the blue and black paths denote the routes walked by walkers from the two bases, respectively. The roundabout path inside the dashed box is unreasonable
In the algorithm of a random walk with intersection, it is assumed that walkers from each side cannot predict the location of the opposite. In practice, locations of the base $v_{s}$ and $v_{e}$ could be obtained in advance, so that walkers from two sides could use the information of their target locations. Then, the algorithm of target guiding self-avoiding random walk with intersection (TGSARWI) is proposed, whose transition probability is deduced as follows.

Assuming that the coordinate of each node is known in the grid network $G$, and the coordinate of node $v_{i}$ is $\left(x_{i}, y_{i}\right)$. If $v_{i}$ and $v_{j}$ are two nodes in the network, the ray angle $\theta_{i j}$ from $v_{i}$ to $v_{j}$ is described as Eq. (7) and Fig. 4.

$$
\theta_{i j}=\left\{\begin{array}{cl}
\arctan \left(\frac{y_{j}-y_{i}}{x_{j}-x_{i}}\right) & \left(y_{j}-y_{i}\right) \arctan \left(\frac{y_{j}-y_{i}}{x_{j}-x_{i}}\right)>0 \\
\pi+\arctan \left(\frac{y_{j}-y_{i}}{x_{j}-x_{i}}\right) & \left(y_{j}-y_{i}\right) \arctan \left(\frac{y_{j}-y_{i}}{x_{j}-x_{i}}\right)<0
\end{array}\right.
$$

When a walker whose destination is $v_{*}\left(x_{*}, y_{*}\right)$ reaches a node $v_{t}\left(x_{t}, y_{t}\right)$ through the route $H_{t}$, its undirected transition probability $p_{t j}^{(1)}\left(H_{t}\right)$ from $v_{t}$ to $v_{j}$ could be calculated by Eq. (6). Since locations of $v_{t}$ and $v_{j}$ have been known in advance, the transition probability could be represented by a vector $\vec{d}_{t j}$ as follows:

$$
\vec{d}_{t j}=p_{t j}^{(1)}\left(H_{t}\right)\left(\cos \theta_{t j}, \sin \theta_{t j}\right)
$$

Meanwhile, unit vector $\overrightarrow{e_{t *}}$ from $v_{t}$ to $v_{*}$ is

$$
\overrightarrow{e_{t *}}=\left(\cos \theta_{t *}, \sin \theta_{t *}\right)
$$

Thus, considering the target guiding, the transition probability can be modified through vector projection as follows: 

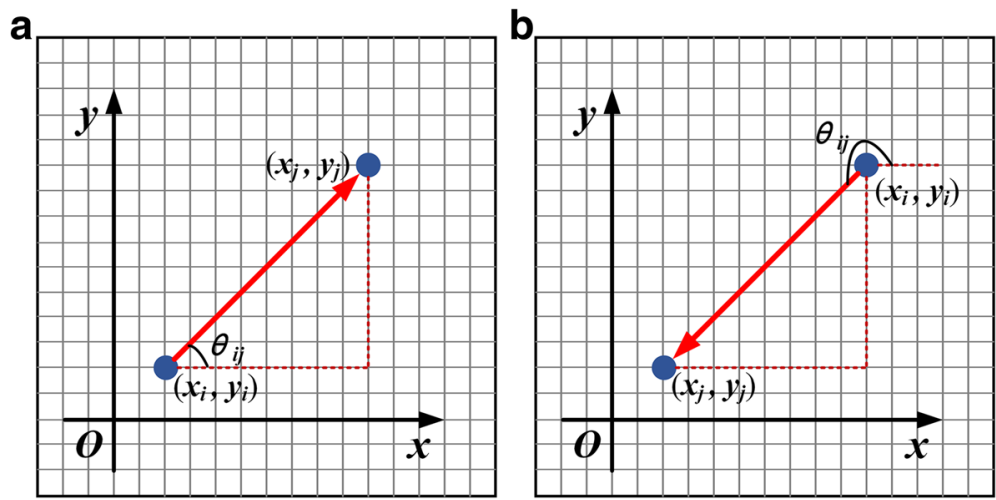

Fig. 4 The diagram of the ray angle $\theta_{i j}$ from $v_{i}$ to $v_{j}$, where the $\theta_{i j}$ in $\mathbf{a}$ is a acute angle, and the $\theta_{i j}$ in $\mathbf{b}$ is a obtuse angle

$$
p_{t j}^{(2)}\left(H_{t}\right)=\overrightarrow{d_{t j}} \cdot \overrightarrow{e_{t *}}=p_{t j}^{(1)}\left(H_{t}\right) \cos \left(\theta_{t j}-\theta_{t *}\right)
$$

It is noted that the value of transition probability $p_{t j}^{(2)}\left(H_{t}\right)$ may be negative. To ensure it satisfies the non-negative and normalization requirement, Eq. (10) is improved as follows:

$$
p_{t j}^{(3)}\left(H_{t}\right)=\frac{p_{t j}^{(1)}\left(H_{t}\right) e^{\rho \cos \left(\theta_{t j}-\theta_{t *}\right)}}{\sum_{\left(v_{t}, v_{k}\right) \in E} p_{t k}^{(1)}\left(H_{t}\right) e^{\rho \cos \left(\theta_{t k}-\theta_{t *}\right)}}
$$

As is shown above, the transition probability of TGSARWI, where $\rho(\rho \geq 0)$ is a target guiding factor, represents the strength of target guiding. When $\rho=0$, the target guiding is ineffective, and $p_{t j}^{(3)}\left(H_{t}\right)=p_{t j}^{(1)}\left(H_{t}\right)$. While $\rho \rightarrow$ $+\infty$, the exposure $w_{t j}$ of the transition edge will play little role to $p_{t j}^{(3)}\left(H_{t}\right)$, and the path found by the algorithm is approximately a straight line between the two bases.

\subsection{Dijkstra algorithm based on sub-network}

Based on random walk algorithm, the TGSARWI algorithm would not definitely produce a connected path which is the optimum one. Thus, $n_{h}\left(n_{h} \gg 1\right)$ connected paths were firstly generated. Then, those paths with the same source point and target point were combined to construct a connected sub-network $G_{1}=\left(V_{1}, E_{1}, W_{1}\right)$ of the grid network $G$ followed by Dijkstra algorithm (DA) to solve MEP problem in this sub-network [44].

The exposure value of one path $H=\left\{v_{t_{1}}, v_{t_{2}}, \ldots, v_{t_{p}}\right\}$ is the sum of all edges' exposures along the path defined as follows:

$$
E(H)=\sum_{j=1}^{p-1} w_{t_{j} t_{j+1}}
$$

DA is considered as a perfect method to solve the shortest path problem of a single source in a non-negative weight network. The core idea of DA is that the generation of the new shortest path is based on the existing shortest. Traditional DA has the shortage that it extends only one node by the shortest tentative distance at each step [45]. In the complex network of grid-based MEP problem, there is a high probability that multiple nodes have the same shortest tentative distance at one step. Thus, DA is improved to allow the extending of multiple nodes at one step and record necessary information.

Assuming that $v_{s}$ and $v_{e}$ are the source and target node, respectively, what the researcher needs to do is to find the shortest path between $v_{s}$ and $v_{e}$. Here, the weight of edge $e_{i j} \in E_{1}$ is defined as $w_{i j}$ in the sub-network $G_{1}$. In DA, $P$ and $T$ are denoted as the sets of permanent and temporary nodes, respectively. Based on the above analysis, the improved DA could be described as follows:

(1) Label $P$ to the node $v_{s}$ and record $P\left(v_{s}\right)=0$. Then, define the set of new nodes labeled $P$ as $R=\left\{v_{s}\right\}$. Label $T$ to other nodes and record $\Delta=\left\{v_{j} \mid v_{j} \in V \& v_{j} \neq v_{s}\right\}$, when $\forall v_{j} \in \Delta, T\left(v_{j}\right)=\infty$;

(2) For each $v_{t} \in R$, for each $v_{j} \in \Delta \& e_{t j} \in E_{1}$, if $T\left(v_{j}\right)>$ $P\left(v_{t}\right)+w_{t j}$, set $T\left(v_{j}\right)=P\left(v_{t}\right)+w_{t j}$ and $\Gamma\left(v_{j}\right)=v_{t} ;$

(3) Set $R=\varnothing$;

(4) For each $v_{i} \in \Delta$, if $T\left(v_{i}\right)=\min _{v_{j} \in T} T\left(v_{j}\right) v_{i} \neq v_{e}$, label $P$ to the node $v_{i}$, and define $P\left(v_{i}\right)=T\left(v_{i}\right), R=R \cup\left\{v_{i}\right\}, \Delta=\Delta$ - $\left\{v_{i}\right\}$. if $T\left(v_{i}\right)=\min _{v_{j} \in \Delta} T\left(v_{j}\right) v_{i}=v_{e}$, label $P$ to the node $v_{i}$, and define $P\left(v_{i}\right)=T\left(v_{i}\right)$, algorithm end, else return to (2).

In the above steps, $\Gamma$ records the shortest path information from the source node to other nodes in the improved DA. The information recorded by $\Gamma$ is incomplete, because the algorithm calculates part of the shortest paths from the source node to all other nodes. However, it can be ensured that the information of the shortest path $H_{m}$ from $v_{s}$ to $v_{e}$ is complete. Then, $H_{m}$ can be obtained through the following backtracking algorithm: 
(1) Initialize $H_{m}=\left\{\Gamma\left(v_{e}\right), v_{e}\right\}$ and set $v_{t}=\Gamma\left(v_{e}\right)$;

(2)If $v_{t}=v_{s}$, backtracking end, or go to (3);

(3) Set $H_{m}=\left\{\Gamma\left(v_{t}\right)\right\} \tilde{\cup} H_{m}, v_{t}=\Gamma\left(v_{t}\right)$, and return to (2).

Where $H_{m}$ is the shortest path from $v_{s}$ to $v_{e}$, which is an ordered set, $\tilde{u}$ in step (3) represents ordered union operation. For example, if there exists an ordered set $A=\left\{v_{1}, v_{2}\right\}$, then the following results will be obtained, $\left\{v_{3}\right\} \tilde{\cup} A=\left\{v_{3}, v_{1}, v_{2}\right\}$ and $A \tilde{\cup}\left\{v_{3}\right\}$ $=\left\{v_{1}, v_{2}, v_{3}\right\}$.

\subsection{Algorithm pseudocode}

Here, the pseudocode is presented for the overall algorithm, including the TGSARWI algorithm and the improved DA (called TGSARWI DA).

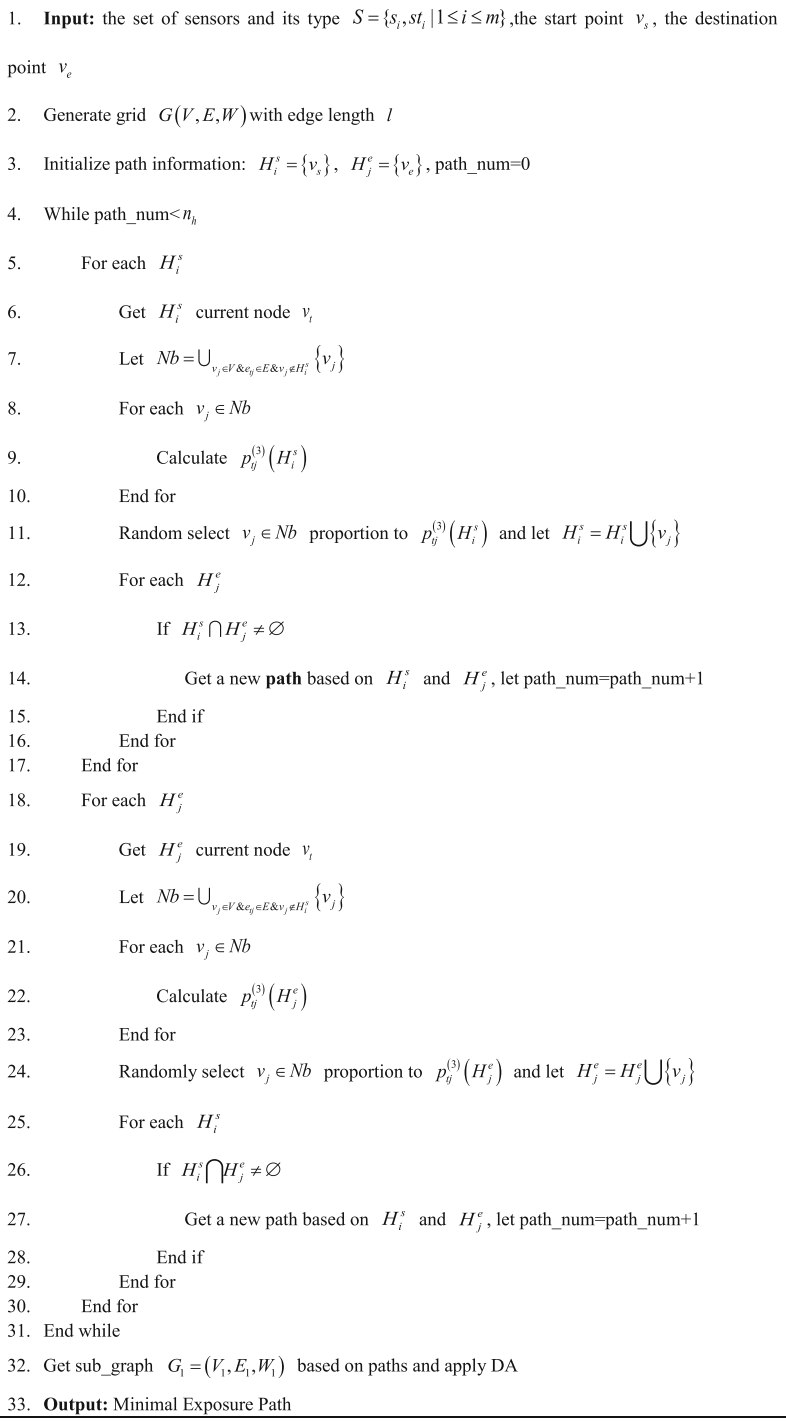

\section{Simulation result and discussion}

The studies about the convergence and complexity for random optimization-based heuristic algorithm are usually based on Markov chain, which has no aftereffect property [46-52]. TGSARWI DA is based on the algorithm of selfavoiding random walk and combines target guiding and intersection of paths. Since the walking process does not have after effect property, it no longer belongs to the category of Markov process. Hence, it is inappropriate to do the complexity analysis for our algorithm by common theories. This work integrates theoretical analysis and numerical simulation to discuss the algorithm performance.

In this section, simulations are conducted to verify the algorithm for the MEP problem. Firstly, the effect of the parameter $\rho$ on the algorithm is evaluated. Secondly, the algorithm performance is analyzed with precision assessment, complexity analysis, and comparison with POA. Finally, the robustness of the algorithm is discussed.

In order to distinguish the application of DA to the global grid network and the sub-network created by TGSARWI, they are defined as Global DA and TGSARWI DA, respectively.

\subsection{Effect evaluation of the target guiding factor}

The effects of the target guiding factor $\rho$ are discussed on the number of iterations for finding the first connected path $\left(T_{f}\right)$, the number of iterations for finding all the required connected paths $\left(T_{l}\right)$, and the minimum exposure $E(H)$, respectively.

The simulation is implemented in the grid with the scale of $50 \times 50$, in which the length of edge is 10 , the number of sensors is 50 and their directions, and coordinates are generated randomly. The initial graph created is shown in Fig. 5. For each parameter $\rho$, the algorithm is simulated 100 times and the average values of $T_{f}, T_{l}$, and $E(H)$ could be obtained.

Figure 6a shows that when the parameter $\rho$ increases, $T_{f}$ and $T_{l}$ decrease. The reason is that the influence of target guiding on the transition probability increases with the increment of $\rho$, which weakens the walker's randomness and decreases the number of iteration. In fact, $T_{f}$ and $T_{l}$ decrease sharply when $\rho$ belongs to the area of $[0,0.9]$. However, when $\rho>0.9$, the numbers of iterations reach stable. This means that, as $\rho$ further increases, the influence on finding the connected path is little.

The effect of parameter $\rho$ to minimum exposure $E(H)$ is displayed in Fig. 6b. Roughly speaking, with the increment of $\rho$, the value of $E(H)$ first decreases and then increases. When $\rho$ is small, the randomness of the walkers is large. Thus, the sub-network formed by $n_{h}$ connected paths is sparse (see Fig. 7a, b), leading to a large value of $E(H)$. However, if $\rho$ is too large, walkers cannot avoid moving along edges with large exposure due to the constraint of target guiding (see Fig. 7c, d). This can also make the value of 


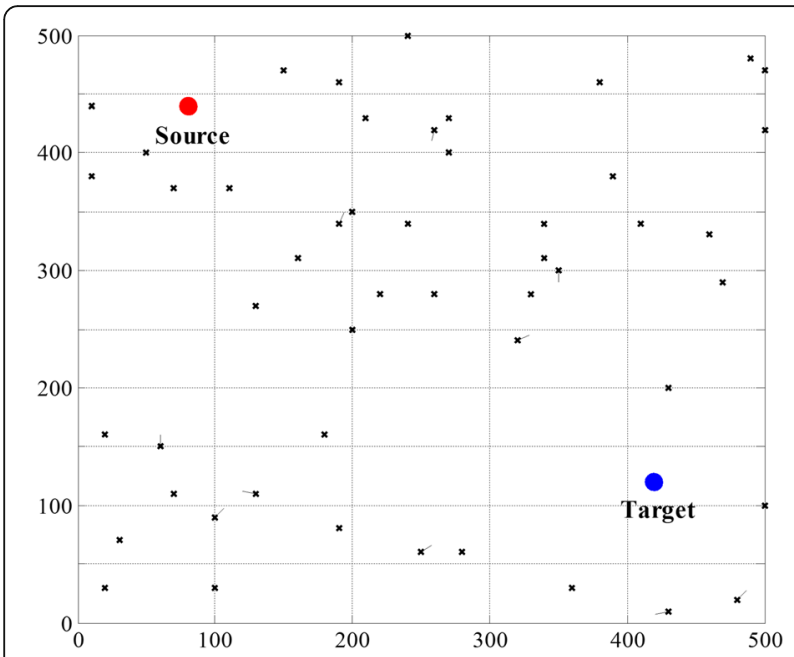

Fig. 5 The initial graph which used to simulate our algorithm, in which sensor types include directional sensor and omnidirectional sensor

$E(H)$ large. Figure 6b shows that $E(H)$ achieves the minimum as $\rho=0.9$. Thus, $\rho$ is set as 0.9 in simulations.

Taking $\rho$ as 0.9 , the MEP problem presented in Fig. 5 is addressed with TGSARWI DA algorithm. It is also solved with Global DA as a comparison. As shown in Fig. 8, although those algorithms generate different optimum paths, the values of $E(H)$ are very close.

It is worth to note that TGSARWI DA algorithm owns good generality. Once the value of parameter $\rho$ is chosen, the algorithm could solve MEP problem with various field scales and different sensor densities. In the following simulations, $\rho$ is always set as 0.9 .

\subsection{Performance evaluation of TGSARWI DA}

The performance of TGSARWI DA algorithm is evaluated from three aspects, i.e., precision, complexity analysis, and comparison with POA.
The minimum exposure path found by Global DA is considered as optimal path. Thus, TGSARWI DA is compared with Global DA. Considering the time efficiency, the first path found by TGSARWI is also included in the comparison. In order to overcome the randomness feature of single simulation, 30 independent repetitive simulations are performed and the average value is taken.

The numbers of walkers and connected paths which form sub-network are related with the scale of the field. As for a field with scale $m \times n$, these values are respectively taken as $n_{u}=\lfloor 30+\sqrt[4]{m n}\rfloor$, and $n_{h}=\lfloor 30+2 \times \sqrt[4]{m n}\rfloor$.

\subsubsection{Precision assessment}

Since the time complexity of Global DA grows rapidly as field scale increases, simulations are initially conducted in the field whose scale changes from $10 \times 10$ to $150 \times 150$ (see Fig. 9a). In order to avoid the effect of sensor density, the density of sensors is fixed as 0.02 . Namely, the sensor number is $2 \%$ of the total number of nodes in the grid network. Then, the field scale is fixed as $50 \times 50$, and the sensor density is adjusted from 0.04 to 0.4 (see Fig. $9 \mathrm{~b}$ ).

Figure 9a shows the trends of $E(H)$ corresponding to the first connected path $H_{\text {TFP }}$ obtained by TGSARWI, path $H_{\mathrm{TDA}}$ obtained by TGSARWI DA and path $H_{\mathrm{GDA}}$ obtained by Global DA with the increasing of scale, respectively. $E(H)$ of $H_{\mathrm{TDA}}$ and $H_{\mathrm{GDA}}$ are almost equal, whose relative error is $4.59 \%$ and maximum value is $6.39 \%$. In contrast, $E(H)$ of $H_{\mathrm{TFP}}$ is much larger than that of $H_{\mathrm{GDA}}$. Their average relative error is $85.49 \%$. In Fig. 9, $E(H)$ of $H_{\mathrm{TDA}}$ and $H_{\mathrm{GDA}}$ are almost the same in various sensor density fields. In fact, their average relative error is only $2.19 \%$. However, $E(H)$ of $H_{\mathrm{TFP}}$ is greater than $H_{\mathrm{GDA}}$, and their average relative error is $50.41 \%$. These results suggest that, when dealing with MEP problem with various field scales and different sensor densities, TGSARWI DA exhibits performance nearly as well as Global DA.
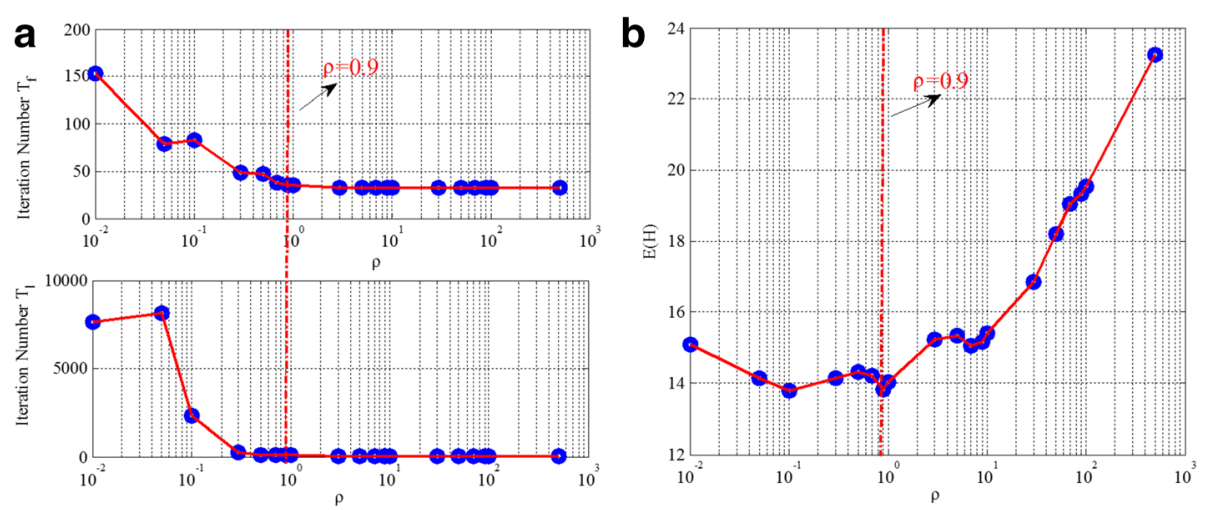

Fig. 6 The diagram of the effect of factor $\rho$ to the algorithm, where a shows the trends of iteration number of first path and TGSARWI paths with the changes of $\rho$, and $\mathbf{b}$ shows the trend of the minimum exposure obtained by TGSARWI DA with the changes of $\rho$ 

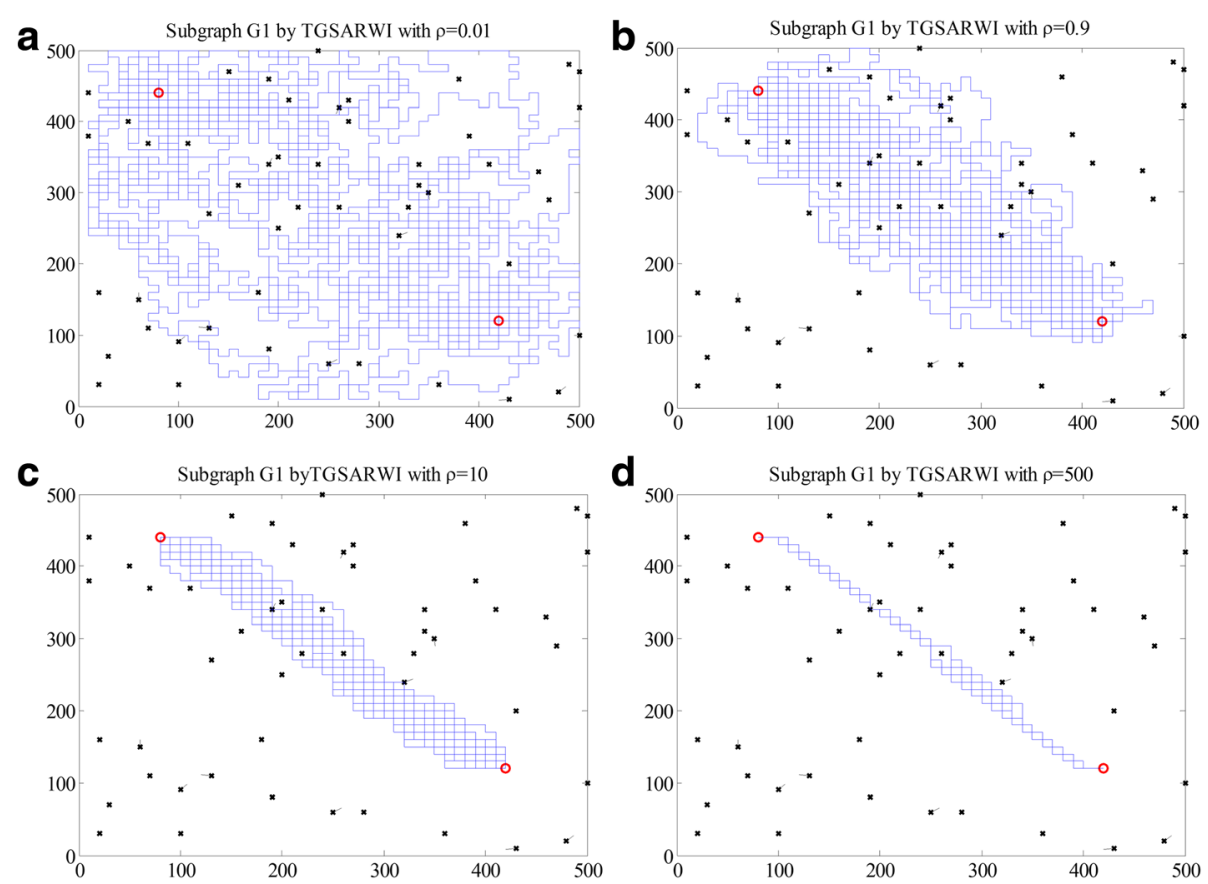

Fig. 7 The sub-network created by TGRWI algorithm with different target guiding factor, where the $\rho$ is set as $0.01,0.9,10$, and 500 , respectively

\subsubsection{Time computation complexity analysis}

For a field with scale $m \times n$, the time computation complexity of DA is $O\left(m^{2} n^{2}\right)$, which means that DA is not suitable for solving MEP problem with a rather largescale field. Here, the time computation complexity of Global DA is compared with TGSARWI DA in different grid scales and various sensor densities. The scale of the field is set from $10 \times 10$ to $900 \times 900$, and the sensor density is in the area of [ $\left[\begin{array}{ll}0 & 0.4\end{array}\right]$.

In this paper, the time computation complexity is measured from two aspects, i.e., first connected path found by

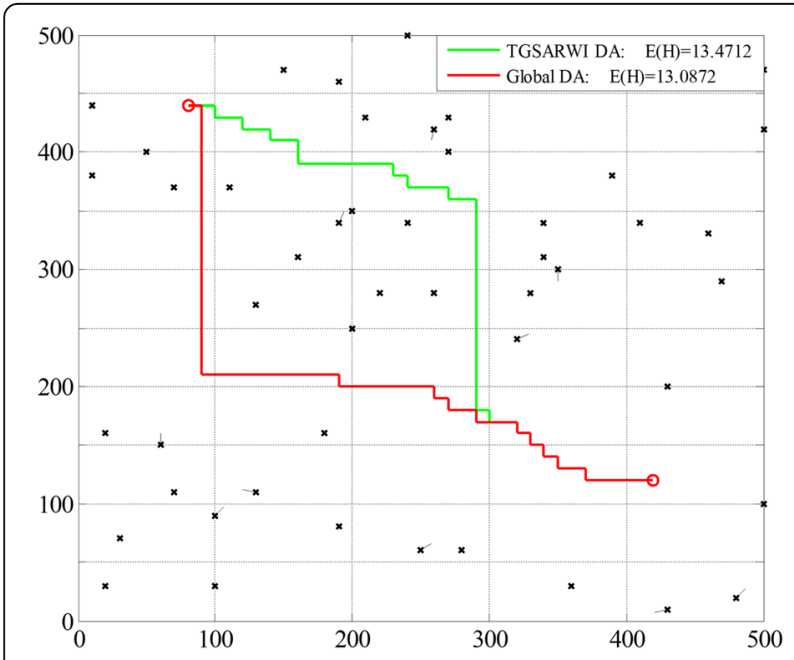

Fig. 8 An example of comparison of Global DA and TGSARWI DA
TGSARWI and the minimum exposure path found by TGSARWI DA.

For the first connected path, the time computation complexity is mainly associated with the transition possibility $p_{t j}^{(3)}\left(H_{t}\right)$ of each walker at each step in the grid network, which is about $O\left(n_{u} \times T_{f}\right)$. Further, the time computation complexity benefit $\eta_{\text {TFP }}$ of the first path found by TGSARWI is defined as the ratio of complexity of finding first connected path by TGSARWI and DA, which is represented as follows:

$$
\eta_{\mathrm{TFP}}=\frac{n_{u} \times T_{f}}{(m \times n)^{2}} \approx T_{f}\left(30(m n)^{-2}+(m n)^{-\frac{7}{4}}\right)
$$

For the minimum exposure path, to quantitatively analyze the simplifying efficacy of TGSARWI algorithm to MEP problem, the reduction ratio $r$ is defined as the norm ratio of sub-network $G_{1}$ formed by $n_{h}$ connected paths to the original grid network $G$ :

$$
r=\frac{\left\|G_{1}\right\|}{\|G\|}=\frac{\left\|G_{1}\right\|}{m \times n}
$$

Where $\|\bullet\|$ is the number of edges of the network. Since the time computation complexity of sub-network forming is about $O\left(n_{u} \times T_{l}\right)$, and the time computation complexity of minimum exposure path found by TGSARWI DA is $O\left(n_{u} \times T_{l}+r^{2} \times(m \times n)^{2}\right)$, the time computation complexity benefit $\eta_{T D A}$ is calculated as follows: 


$$
\eta_{\mathrm{TDA}}=\frac{n_{u} \times T_{l}+r^{2} \times(m \times n)^{2}}{(m \times n)^{2}} \approx T_{l}\left(30(m n)^{-2}+2(m n)^{-\frac{7}{4}}\right)+r^{2}
$$

In Fig. 10a, the iteration number $T_{f}$ is growing linearly as the field scale increases, whose slope is about 0.9803 . Compared with the time computation complexity of DA that is $O\left(m^{2} n^{2}\right)$, the efficiency of first path found by TGSARWI algorithm is higher. From Eq. (13) and Fig. 10b, it is shown that the first path benefit $\eta_{\text {TFP }}$ decreases significantly as the scale increases. Specifically, when the field scale is $900 \times$ $900, \eta_{\text {TFP }}$ is only $8.3493 \times 10^{-8}$. These results suggest that TGSARWI is efficient to find out the first path for timeliness demand in large-scale field, such as real-time solution and online calculation, although the exposure performance of $H_{\mathrm{TFP}}$ is not as good as Global DA.

In Fig. 10c, although the iteration number $T_{l}$ of TGSARWI DA increases exponentially with the change of field scale, its exponential coefficient is only about 0.0046. Meanwhile, in Fig. 10d, the complexity benefit $\eta_{\text {TDA }}$ gradually deceases with the increasing of the scale, and finally goes close to zero. Particularly, its minimum value is 0.0042 when the field has the scale of $900 \times 900$, suggesting that TGSARWI DA only uses $0.42 \%$ computation time of Global DA.

Similar measures are also employed to analyze the time computation complexity of TGSARWI DA with various sensor densities. In Fig. 11a, iteration numbers of the first path found in the field for different sensor densities are visualized. The number of iterations increases linearly with the density of sensors in the areas of $[0.0 .312]$ and $(0.312,0.4]$, respectively. The linear relation suggests that TGSARWI is not sensitive to the change of sensor density, making it suitable to solve MEP problem in the field with high density sensors.
The equations of the two fitting lines are shown in the inner figure. The slope of the straight line in the latter area is much larger than that in the former area, implying that the time to find a path increases sharply when the density of sensors exceeds the critical value 0.312 . This is because if the distribution of sensors is too dense, it will be rather difficult for the walker to find an accessible path. In the area that density of sensors is reasonable (here it is $[0,0.312]$ ), TGSARWI is expected to identify a path at a rather fast speed. For example, when the number of sensors is 780 , corresponding to the density of sensor 0.312 , only 41 steps are needed to find the first connected path. Figure 11b shows how the iteration number to find all the required connected paths changes with sensor density. The iteration number $T_{l}$ is proportional to the sensor density. The curve increases gently when the sensor density is below 0.312 and grows rapidly as the density is above 0.312 . The fitting function of the curve is $y=\frac{30.2064}{0.4276-x}$. Accordingly, it is obviously shown that the complexity of finding connected path increases as the density becomes denser, while the maximum sensor density for TGSARWI algorithm to find connected sub-network is 0.4276 .

\subsection{Comparison with POA}

As a representative of heuristic algorithms in solving MEP problem, physarum optimization algorithm (POA) has the ability to find the minimum exposure path in the field with various type sensors and reduce the time computation complexity [40]. However, the growth of time computation complexity is still too fast.

According to ref. [40], in the field with scales of $10 \times$ $10,20 \times 20,50 \times 50$, and $100 \times 100$, as well as with the sensor number of 10,30 , and 50, the minimum exposures of paths found by POA and DA are quite close. The relative errors of $E(H)$ of paths got by POA and
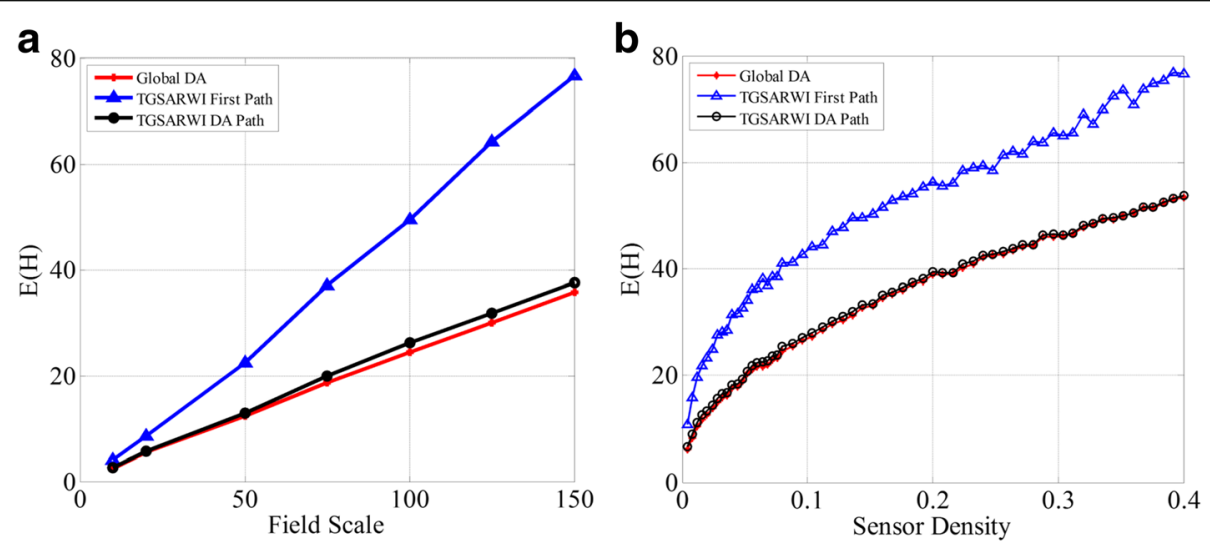

Fig. 9 Precision comparison of Global DA, TGSARWI DA, and the first path obtained by TGSARWI in various field scales and sensor densities, where $\mathbf{a}$ shows the exposure trends of three paths with the changes of field scale, and $\mathbf{b}$ shows the exposure trends of three paths with the changes of sensor density 

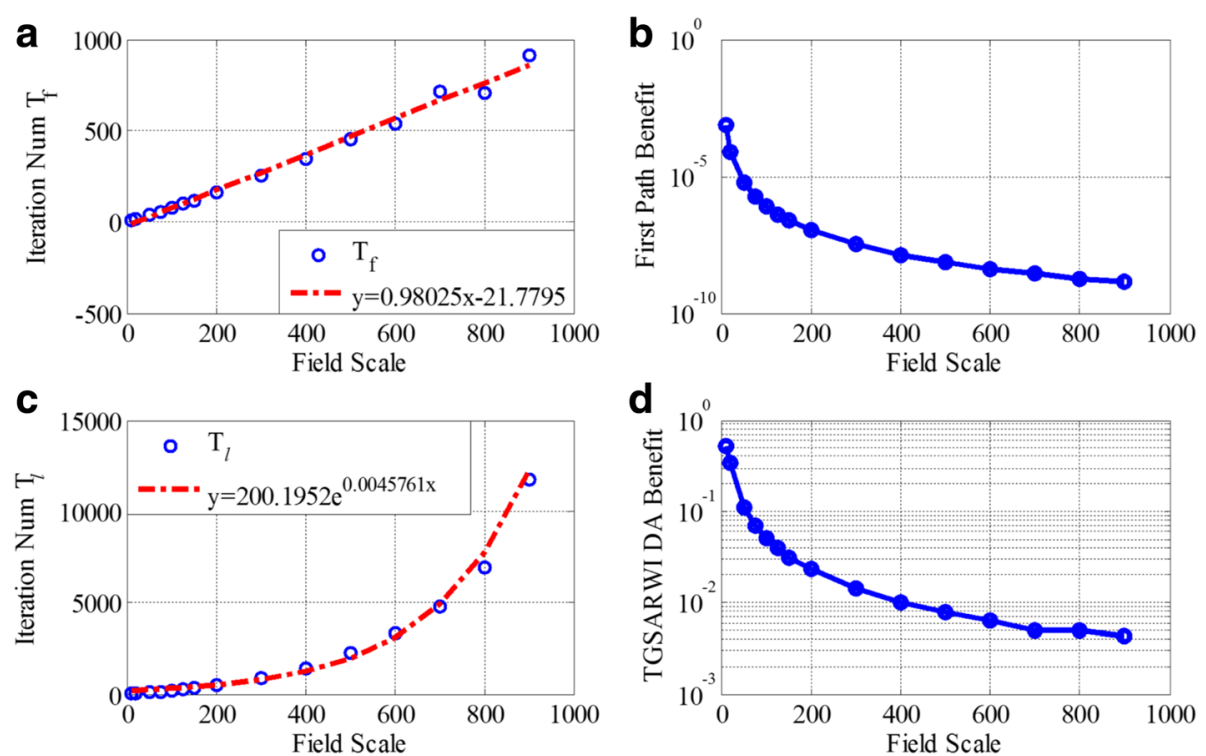

Fig. 10 The time computation complexity of TGSARWI with various field scales. a Shows the trend of iteration number of the first path obtained by TGSARWI. $\mathbf{b}$ Shows the trend of the first path benefit. $\mathbf{c}$ Shows the trend of iteration number for finding all the required connected paths. $\mathbf{d}$ Shows the benefit trend of iteration number for finding all the required connected paths

Global DA are 0.0240 and 0.0542 in different field scales and sensor densities. TGSARWI DA is also compared with Global DA in the same condition. The relative errors are 0.0219 and 0.0459 in different field scales and sensor densities, respectively. These results suggest that the algorithm has slightly better performance than POA.

The time computation complexity of TGSARWI DA is further compared with that of POA. Numbers of iteration for POA are taken from ref. [31]. As shown in Fig. 12, the iteration number of POA is far greater than that of TGSARWI DA, no matter what scale the field and the sensor density are. These results fully demonstrate that TGSARWI DA is more appropriate to solve MEP problem with a large-scale field and high-sensor density.

\subsection{Robustness discussion}

In this section, variation coefficient is applied to quantitatively analyze the vulnerability of TGSARWI deceased by the stochastic fluctuations of target guiding factor $\rho$, the number of walkers $n_{u}$ and the number of connective paths $n_{h}$ [53]. The fluctuation coefficient $\phi(X)$ of sample data $X$ is defined as:

$$
\phi(X)=\frac{\sqrt{D(X)}}{F(X)}
$$

where $F(X)$ and $D(X)$ donate the expectation and variance of $X$, respectively. The closer the coefficient $\phi(X)$ to zero is, the smaller fluctuation the sample data $X$ has.

Based on current parameters, 100 groups of normal distribution rates are generated and 50 groups of sample
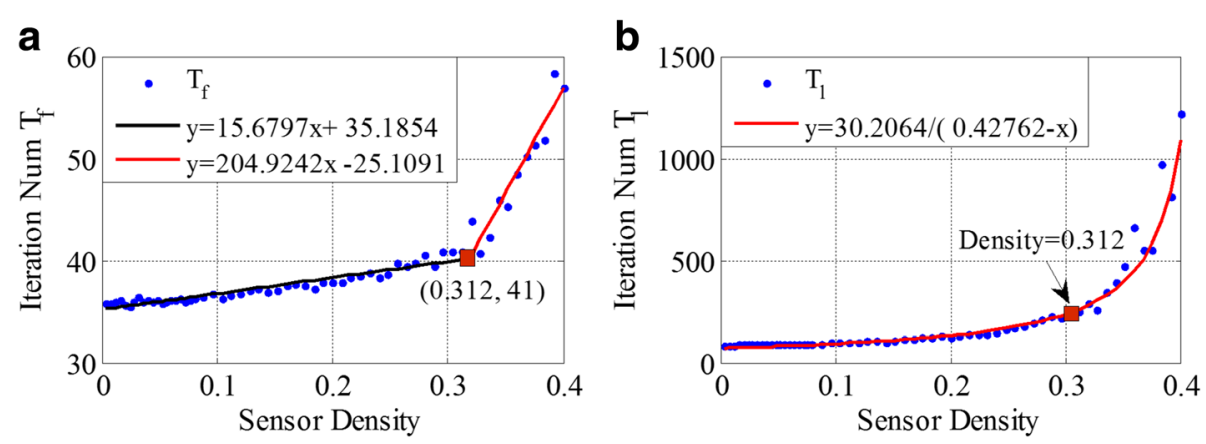

Fig. 11 The time computation complexity of TGRWI for various sensor densities. a Shows the trend of iteration number of the first path obtained by TGSARWI. $\mathbf{b}$ Shows the trend of iteration number for finding all the required connected paths 

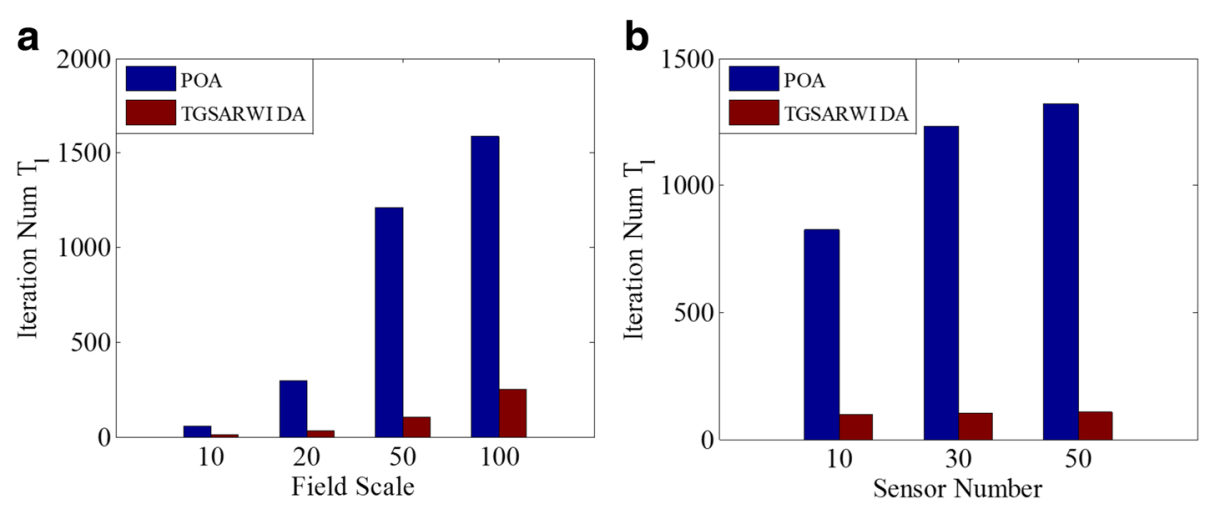

Fig. 12 Comparison of time computation complexity for POA and TGSARWI DA with different field scales and sensor number. a Shows the comparison of iteration number $T_{\text {, with }}$ the various field scales. $\mathbf{b}$ Shows the comparison of iteration number $T_{\text {, with }}$ the different sensor number

data are created, according to each normal distribution rate. Then, corresponding iteration number $T_{l}$, and the minimum exposure $E(H)$ are calculated according to each normal distribution. Finally, values of $\phi(\rho), \phi\left(n_{u}\right)$, $\phi\left(n_{h}\right), \phi\left(T_{l}\right)$, and $\phi(E(H))$ are obtained. $\phi\left(T_{l}\right)$ and $\phi(E(H))$ are set as vertical coordinates, $\phi(\rho), \phi\left(n_{u}\right)$, and $\phi\left(n_{h}\right)$ as horizontal coordinates, respectively and show their relationships in Fig. 13.

From Fig. 13a, b, when $\rho$ and $n_{u}$ fluctuate in the interval $[0,0.5]$, the fluctuation coefficients of the minimum exposure $E(H)$ change little, the maximum values of $\phi(E(H))$ are 0.0331 and 0.0315 , respectively. They are all smaller than the corresponding fluctuation coefficients of parameters, indicating that TSAGRWI DA owns quite excellent robustness to the stochastic fluctuations of these two parameters. In Fig. 13c, $\phi(E(H))$ increases quadratically with $\phi\left(n_{h}\right)$, but still satisfies $\phi(E(H))$ $<\phi\left(n_{h}\right)$. The maximum value of $\phi(E(H))$ is 0.0726 , indicating $E(H)$ also owns better robustness to the stochastic fluctuation of $n_{h}$. In summary, $E(H)$ is not sensitive to the fluctuation of the three parameters $n_{h}, \rho$, and $n_{u}$.

Figure 13d-e show that $\phi\left(T_{l}\right)$ increases as $\phi(\rho), \phi\left(n_{u}\right)$, and $\phi\left(n_{h}\right)$ grow. The values of $\phi\left(T_{l}\right)$ increases quadratically with the increase of $\phi(\rho)$ and $\phi\left(n_{u}\right)$, and the increasing rate of the former is faster than the latter. When $\phi(\rho) \leq 0.1611$ and $\phi\left(n_{u}\right) \leq 0.2370$, the fluctuation coefficient of $T_{l}$ is smaller than those of $\rho$ and $n_{u}$, suggesting that $T_{l}$ is not sensitive to the fluctuation of $\rho$ and $n_{u}$ in these areas. $\phi(\rho)>0.1611$ and $\phi\left(n_{u}\right)>0.2370$, the fluctuation coefficient of $T_{l}$ is larger than those of $\rho$ and

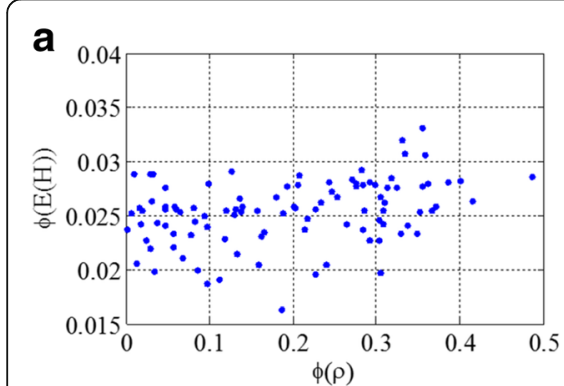

d

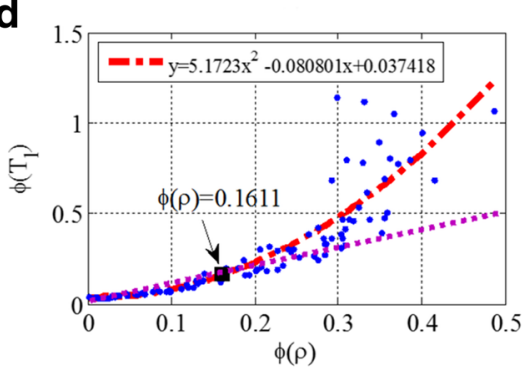

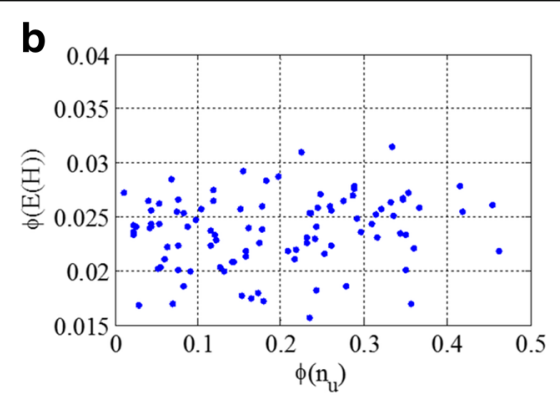

e

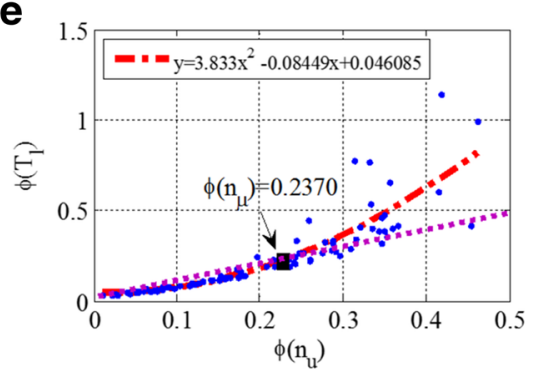

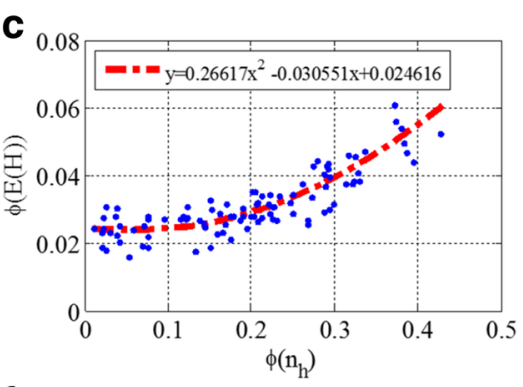

f

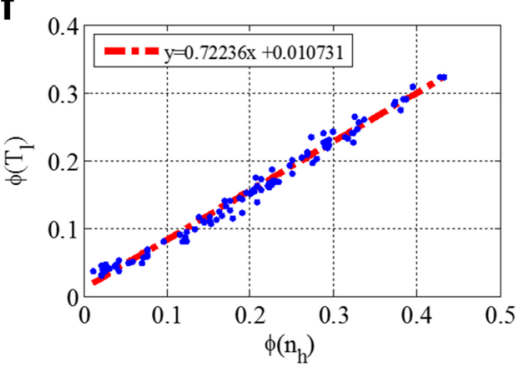

Fig. 13 Comparisons of fluctuation coefficients of the parameters. a Shows the fluctuation effect of $\rho$ to $E(H)$. $\mathbf{b}$ Shows the fluctuation effect of $n_{u}$ to $E(H)$. c Shows the fluctuation effect of $n_{h}$ to $E(H)$. $\mathbf{d}$ Shows the fluctuation effect of $\rho$ to $T_{1}$. e Shows the fluctuation effect of $n_{u}$ to $T_{1}$. $\mathbf{f}$ Shows the fluctuation effect of $n_{h}$ to $T_{1}$ 
$n_{u}$, which indicates that $T_{l}$ is sensitive to the fluctuation of $\rho$ and $n_{u}$ in these areas. $\phi\left(T_{l}\right)$ grows linearly as $\phi\left(n_{h}\right)$ increases, and the slope is 0.7224 , which is smaller than 1 , implying that $T_{l}$ has better robustness to the fluctuation of $n_{h}$. In a word, the sensitiveness of iteration number $T_{l}$ to the stochastic fluctuations of parameters could be sorted in descending order as $\rho, n_{u}$, and $n_{h}$.

It is worth to note that both fluctuation coefficients of $T_{l}$ and $E(H)$ have positive values during the fluctuations of $\rho, n_{u}$, and $n_{h}$, which are mainly caused by the randomness of TGSARWI.

\section{Conclusions}

MEP problem comes from the requirement to evaluate the coverage quality of field with sensor deployment. According to the minimum exposure path, the deployment of sensors could be improved. This study aims to enhance the computation efficiency for MEP problem. The random walk is modified from the perspective of target guiding, selfavoiding, and route interaction to propose an algorithm called TGSARWI. A sub-network is constructed by multiple connected paths generated by a group of random walkers using TGSARWI. Then, Dijkstra algorithm is applied to solve MEP problem in this sub-network. The simulations mentioned in this study suggest that, the minimum exposure path solved by the approach above is comparable to that solved by Global DA, while the time computation complexity of TGSARWI DA is only $10^{-3}$ of that for DA. Compared with existing heuristic algorithms such as physarum optimization algorithm (POA), this algorithm exhibits higher generality and efficiency. Therefore, our algorithm could solve MEP problem in fields with largescale or high-density sensors. In fact, it is possible to extend TGSARWI DA to solve the MEP problem in the threedimensional field, or the field with special protection area, etc. It is expected to shed new lights on the study about MEP problem and promote the development of WSN.

\section{Endnotes}

${ }^{1}$ Note: There also exist some improved Dijkstra's algorithm. For example, the reference [54] uses special data structure, Fibonacci heaps, to improve the efficiency of search and comparison, and then it makes the time computation complexity decrease to $O(E+\log V)$, where $E$ and $V$ represent the numbers of edge and node in the graph $G(V, E)$, respectively.

\section{Abbreviations}

DA: Dijkstra algorithm; MEP: Minimum exposure path; POA: Physarum optimization algorithm; QoS: Quality of service; TGSARWI: Target guiding selfavoiding random walk with intersection; WSN: Wireless sensor network

\section{Funding}

This work was partially supported by the National Natural Science Foundation of China under Grant 61372194, Grant 61672038, Grant 70871119, Grant 61502520, Grant 10971227, and Grant 21377166, Excellent Teaching Team Building Project of Logistics Engineering University in 2014, 2110 Engineering Construction Project Phase III, Chongqing Education Reform Project of Graduate under Grant yjg152017, and the Graduate Student Research and Innovation Funding Project of Chongqing in China under Grant CYB16129.

\section{Availability of data and materials}

Not applicable

\section{Authors' contributions}

In this research paper, D- $\sqcup$ and JZ devised and guided the research project. T-HY and H.-YF proposed the algorithm. T-HY wrote programs. MT and LX analyzed the results. H-YF wrote the paper. All authors have read and approved the final manuscript.

\section{Authors' information}

T.-H.Y. is currently working toward the PhD degree in Institute of Modern Logistics, Logistical Engineering University, China. He received the MS degree in Communication Engineering from Southwest Electronic Telecommunication Research Institute, China, in 2005, and the BS degree in Applied Mathematics from School of science, National University of Defense Technology, China, in 2002. He is an associate professor of Department of Fundamental, Logistical Engineering University, China. His research interests include the fields of Internet of things and multimedia sensor networks.

D.-L.J. received the PhD degree in Traffic management engineering from the School of Transportation and Logistics, Southwest Jiao Tong University,

China, in 1998, the MS degree in material flow engineering from Institute of Modern Logistics, Logistical Engineering University, China, in 1990, and the

BS degree in automatic control engineering from National University of Defense Technology, China, in 1984. He is a professor and director of Institute of Modern Logistics, Logistical Engineering University, China. He visited University of Missouri at Saint Louis as a research fellow in 2009 and 2010, respectively. His current research include optimization theory and method, logistics and supply chain management, and internet of things, and he has published over 100 papers and seven books on these fields. H.-Y.F. is currently working toward the PhD degree in Institute of Modern Logistics, Logistical Engineering University, China. His research interests include the fields of operational research and optimization theory. M.T. is currently working toward the PhD degree in Institute of Modern Logistics, Logistical Engineering University, China. His research interests include the fields of operational research, complex network and optimization theory.

L.X. received the MS degree in control theory and control engineering from the School of automation, Chongqing University, China, in 2009, and the BS degree in electronic information science and technology from Department of physics and information technology, Chongqing Normal University, China, in 2003. She is an associate professor and director of Department of Information Engineering, Chongqing Electric Power College, China. Her research interests include the fields of Internet of things, electronic information and automatic control. J.Z. received the PhD degree in Biomedical engineering from College of Life Science and Technology, Shanghai Jiao Tong University, China, in 2007, the MS degree in Applied Mathematics from School of Mathematics and Statistics, Chongqing University, China, in 1988. She is a professor and director of Department of Mathematics, Logistical Engineering University, China. Her current research include optimization theory and method, complex network, and network pharmacology, and she has published over 80 papers and five books on these fields.

\section{Competing interests}

The authors declare that they have no competing interests.

\section{Publisher's Note}

Springer Nature remains neutral with regard to jurisdictional claims in published maps and institutional affiliations. 


\section{Author details}

'Department of Military Logistics, Army Logistics University, Chongqing, China. ${ }^{2}$ Department of Mathematics, Army Logistics University, Chongqing, China. ${ }^{3}$ Department of Information Engineering, Chongqing Electric Power College, Chongqing 401311, China.

Received: 24 July 2017 Accepted: 19 January 2018

Published online: 06 February 2018

\section{References}

1. FL Lewis, Wireless sensor networks. Smart Environments Technologies Protocols \& Applications 181(1), 11-46 (2016)

2. V Potdar, A Sharif, E Chang, Wireless sensor networks: a survey. Comput. Netw. 38(4), 393-422 (2009)

3. P Rawat, KD Singh, H Chaouchi, JM Bonnin, Wireless sensor networks: a survey on recent developments and potential synergies. J. Supercomput. 68(1), 1-48 (2014)

4. $\quad V$ Peiris, Highly integrated wireless sensing for body area network applications. SPIE Newsroom., 2-4 (2013)

5. TO Donovan, JO Donoghue, C Sreenan, D Sammorr, PO Reilly, KAO Connor, A Context Aware Wireless Body Area Network (BAN), Pervasive Computing Technologies for Healthcare Pervasivehealth (2009), pp. 1-8

6. B Liu, Z Yan, MAC CW Chen, Protocol in wireless body area networks for E-health: challenges and a context-aware design. IEEE Wirel. Commun. 20(4), 64-72 (2013)

7. JK Hart, K Martinez, Environmental sensor networks: a revolution in the earth system science? Earth Sci. Rev. 78(3-4), 177-191 (2006)

8. P Balaya, in Survey of Energy Harvesting and Energy Scavenging Approaches for on-Site Powering of Wireless Sensor- and Microinstrument-Networks. International Society for Optics and Photonics (California, 2013), p. 87280 S

9. A Tiwari, P Ballal, FL Lewis, Energy-efficient wireless sensor network design and implementation for condition-based maintenance. Acm Transactions on Sensor Networks. 3(1), 1 (2007)

10. K Saleem, N Fisal, J Al-Muhtadi, Empirical studies of bio-inspired selforganized secure autonomous routing protocol. IEEE Sensors J. 14(7), 2232-2239 (2014)

11. G Anastasi, O Farruggia, G Lo Re, M Ortolani, International Conference on System Sciences, Monitoring high-quality wine production using wireless sensor networks (Hawaii, 2009), pp. 1-7

12. J Liang, M Liu, X Kui, A survey of coverage problems in wireless sensor networks. Sensors \& Transducers. 163(1), 240-246 (2014)

13. D Tao, TY Wu, A survey on barrier coverage problem in directional sensor networks. Sensors Journal IEEE. 15(2), 876-885 (2015)

14. C Zhu, C Zheng, L Shu, G Han, A survey on coverage and connectivity issues in wireless sensor networks. Journal of Network \& Computer Applications. 35(2), 619-632 (2012)

15. A Nayyar, S Sharma, S SAnand Nayyar, A survey on coverage and connectivity issues surrounding wireless sensor network. 89 suppl 4(37), 225 (2014)

16. K Hirani, PM Singh, A survey on coverage problem in wireless sensor network. International Journal of Computer Applications. 116(2), 1-3 (2015)

17. J CHou, DKY Yau, CYT Ma, Y Yang, H Zhang, IH Hou, NSV Rao,M Shankar, Coverage in Wireless Sensor Networks. 3(5), 47-79 (2009)

18. S Megerian, F Koushanfar, M Potkonjak, MB Srivastava, Worst and best-case coverage in sensor networks. IEEE Trans. Mob. Comput. 4(1), 84-92 (2005)

19. P Balister, B Bollobás, A Sarkar, Barrier coverage. Random Struct. Algoritm. 49(3), 429-478 (2016)

20. Z Wang, J Liao, Q Cao, H Qi, Z Wang, Barrier coverage in hybrid directional sensor networks. IEEE Trans. Mob. Comput. 13(7), 1443-1455 (2014)

21. T Clouqueur, V Phipatanasuphorn, P Ramanathan, KK Saluja, In Proceedings of the 1st ACM international workshop on Wireless sensor networks and applications, Sensor Deployment Strategy for Target Detection (GA, 2003), pp. 42-48

22. L Zhang, X Chen, J Fan, D Wang, Seventh International Symposium on Parallel Architectures, Algorithms and Programming, The Minimal Exposure Path in Mobile Wireless Sensor Network (Nanjing, 2015), pp. 73-79

23. XY Li, PJ Wan, O Frieder, Coverage in wireless ad hoc sensor networks. IEEE Trans. Comput. 52(6), 753-763 (2003)

24. S Megerian, F Koushanfar, G Qu, G Veltri, M Potkonjak, Exposure in wireless sensor networks: theory and practical solutions. Wirel. Netw 8(5), 443-454 (2002)
25. J Chang, J Yu, J Ke, J Hu, International Conference on Information NETWORKING and Automation, Simulation of worst and best-case coverage for wireless sensor network (Kunming, 2010), pp. 291-295

26. G Veltri, Q Huang, G Qu, M Potkonjak, International Conference on Embedded Networked Sensor Systems, Minimal and maximal exposure path algorithms for wireless embedded sensor networks (California, 2003), pp. 40-50

27. IM Gelfand, SV Fomin, Calculus of variations. Progress in nonlinear differential equations and their applications 12(3), 141-183 (1963)

28. AH Siddiqui, Applied Functional Analysis. E. Horwood, (1980)

29. HN Djidjev, Approximation algorithms for computing minimum exposure paths in a sensor field. Acm Transactions on Sensor Networks. 7(3), 23 (2010)

30. HN Djidjev, Third IEEE International Conference of Distributed Computing in Sensor Systems, Efficient computation of minimum exposure paths in a sensor network field (NM, 2007), pp. 295-308

31. L Liu, X Zhang, H Ma, Global Telecommunications Conference, Minimal exposure path algorithms for directional sensor networks (Hawaii, 2009), pp. 6155-6160

32. J Zhou, J Wen, H Zhang, L Zhang, A nodal integration and post-processing technique based on Voronoi diagram for Galerkin meshless methods. Comput. Methods Appl. Mech. Eng. 192(35), 3831-3843 (2003)

33. W Zhang, M Li, W Shu, MY Wu, Second International Conference of Mobile Ad-hoc and Sensor Networks, Smart Path-Finding with Local Information in a Sensory Field (Hong Kong, 2006), pp. 119-130

34. S Meguerdichian, S Slijepcevic, V Karayan, M Potkonjak, Proceedings of the 2nd ACM International Symposium on Mobile Ad hoc networking and computing, Localized Algorithms in Wireless Ad-Hoc Networks: Location Discovery and Sensor Exposure (New York, 2001), p. 106

35. L Liu, Y Song, H Zhang, H Ma, AV Vasilakos, Physarum optimization: A biology-inspired algorithm for the Steiner tree problem in networks. IEEE Trans. Comput. 64(3), 819-832 (2015)

36. X Liu, G Kang, N Zhang, Percolation theory-based exposure-path prevention for 3D-wireless sensor networks coverage. Ksii Transactions on Internet \& Information Systems. 9(1), 126-148 (2015)

37. H Feng, L Luo, Y Wang, M Ye, R Dong, A novel minimal exposure path problem in wireless sensor networks and its solution algorithm. International Journal of Distributed Sensor Networks. 12(8) (2016)

38. L Liu, G Han, H Wang, J Wan, Obstacle-avoidance minimal exposure path for heterogeneous wireless sensor networks. Ad Hoc Netw. 55, 50-61 (2016)

39. L Wang, T Wang, Dynamic heuristic anti-monitoring path searching algorithm in sensory field. Journal of Computer Applications. 28(11), 2767-2770 (2008)

40. L Liu, Y Song, H Ma, X Zhang, INFOCOM of Proceedings IEEE, Physarum optimization: a biology-inspired algorithm for minimal exposure path problem in wireless sensor networks (Orlando, 2012), pp. 1296-1304

41. $M$ Ye, Y Wang, C Dai, X Wang, A hybrid genetic algorithm for the minimum exposure path problem of wireless sensor networks based on a numerical functional extreme model. IEEE Trans. Veh. Technol. 65, 1-1 (2015)

42. G Kang, X Liu, N Zhang, Y Guo, F Labeau, Critical density for exposure-path prevention in three-dimensional wireless sensor networks using percolation theory. International Journal of Distributed Sensor Networks. 2015(1-12) (2015)

43. F Spitzer, Principles of random walk. J. R. Stat. Soc. 73(128), 563 (1964)

44. A DE Knuth, Generalization of Dijkstra's algorithm. Inf. Process. Lett. 6(1), 1-5 (1977)

45. LW Wayne, Operations research: applications and algorithms. Duxbury Press. (1994)

46. G Rudolph, Convergence analysis of canonical genetic algorithms. IEEE Trans. Neural Netw. 5(1), 96-101 (2002)

47. G Rudolph, Finite Markov chain results in evolutionary computation: a tour d'Horizon. Fundamenta Informaticae 35(1-4), 67-89 (1998)

48. G Rudolph, in Evolutionary Computation, IEEE World Congress on Computational Intelligence., Proceedings of the First IEEE Conference on 1994. Convergence of non-elitist strategies, vol 61 (1994), pp. 63-66

49. G Rudolph, Convergence of evolutionary algorithms in general search spaces. IEEE International Conference on. Evolutionary Computation, 50-54 (1996)

50. J He, X Yu, Conditions for the convergence of evolutionary algorithms. J. Syst. Archit. 47(7), 601-612 (2001)

51. G Rudolph, How mutation and selection solve long-path problems in polynomial expected time. Evol. Comput. 4(2), 195-205 (2014)

52. $\mathrm{Y} Y \mathrm{u}, \mathrm{ZH}$ Zhou, A new approach to estimating the expected first hitting time of evolutionary algorithms. Artif. Intell. 172(15), 1809-1832 (2008)

53. TS Breusch, AR Pagan, A simple test for heteroscedasticity and random coefficient variation. Econometrica 47(5), 1287-1294 (1979)

54. ML Fredman, Fibonacci heaps and their uses in improved network optimization algorithms. J. ACM 34(3), 596-615 (1987) 